\title{
The characteristics of stroke units in Ontario: a pan-provincial survey
}

Valeria E. Rac ${ }^{1,2^{*}}$, Yeva Sahakyan ${ }^{1}$, Iris Fan ${ }^{1}$, Luciano leraci ${ }^{3}$, Ruth Hall ${ }^{2,4,5}$, Linda Kelloway ${ }^{5}$, Gabrielle van der Velde ${ }^{1,6}$, Moira K. Kapral ${ }^{2,4,7,8}$, Mark Bayley ${ }^{2,4,8,9}$ and Murray Krahn ${ }^{1,2,4,6,7,8}$

\begin{abstract}
Background: Previous studies have demonstrated that organized, multidisciplinary care is the cornerstone of current strategies to reduce the death and disability caused by stroke. Identification of stroke units and an understanding of their composition and operation would provide insight for the further actions required to improve stroke care. The objective of this study was to identify and survey stroke units in Canada's largest province, Ontario (population of 13 million) in order to describe availability, structure, staffing, processes of care, and type of population stroke units serve.
\end{abstract}

Methods: The Ontario Stroke Network (2011) list of stroke units and snowball sampling was used to identify all stroke units. During 2013 - 2014 an interviewer conducted telephone surveys with the stroke unit managers using closed and semi-open ended questions. Descriptive statistics were used to summarize survey responses.

Results: The survey identified 32 stroke units, and a respondent from every stroke unit (100\% response rate) was interviewed. Twenty one were acute stroke units, 10 were integrated stroke units and one was classified as a rehabilitation stroke unit. Stroke units were available in all 14 Local Health Integration Networks except Central West. The estimated average number of stroke patients served per stroke unit was 604 with six-fold variation (242 to 1480) across the province. The typical population served in stroke units were patients with either ischemic or hemorrhagic stroke. Data consistently reported on the processes of stroke care, including the availability of multidisciplinary staff, specific diagnostic imaging, use of validated assessment tools, and the delivery of patient education. Details about the core components of stoke care were provided by 16 stroke units (50\%).

Conclusions: This study demonstrates the heterogeneous structure of stroke units in Ontario and signaled potential disparity in access to stroke units. Many core components are in place, but half of the stroke units in Ontario do not meet all criteria. Areas for potential improvement include stroke care training for the multidisciplinary team, provision of individualized rehabilitation plans, and early discharge assessment.

Keywords: Stoke units, Core component, Environmental scan, Ontario

\footnotetext{
*Correspondence: valeria.rac@theta.utoronto.ca

'Toronto Health Economics and Technology Assessment (THETA)

Collaborative, Toronto General Research Institute, Toronto General Hospital,

University Health Network, University of Toronto, Toronto, ON, Canada

${ }^{2}$ Institute of Health Policy, Management and Evaluation (IHPME), University of

Toronto, Toronto, ON, Canada

Full list of author information is available at the end of the article
}

\section{Ciömed Central}

(c) The Author(s). 2017 Open Access This article is distributed under the terms of the Creative Commons Attribution 4.0 International License (http://creativecommons.org/licenses/by/4.0/), which permits unrestricted use, distribution, and reproduction in any medium, provided you give appropriate credit to the original author(s) and the source, provide a link to the Creative Commons license, and indicate if changes were made. The Creative Commons Public Domain Dedication waiver (http://creativecommons.org/publicdomain/zero/1.0/) applies to the data made available in this article, unless otherwise stated. 


\section{Background}

Stroke is a major health challenge, placing a substantial burden on patients, families and health care systems. Each year 15 million people suffer a stroke worldwide, where nearly six million die and another five million experience long-term disability [1]. In Canada, stroke is the third leading cause of death [1]. It imposes a significant cost to the Canadian economy with $\$ 3.6$ billion spent annually in hospital and physician services, lost wages, and decreased productivity [1]. Due to the aging population, the absolute number of individuals at risk is projected to increase [2]. According to the World Health Organization, disability-adjusted life years lost to stroke will increase from 38 to 61 million between 1990 and 2020 [3].

Organized care is the cornerstone of current strategies to reduce mortality and disability caused by stroke. Randomized controlled clinical trials have shown a reduction in mortality, dependency and institutionalized care for patients treated in stroke units (SUs) relative to patients who received care in general wards $[4,5]$. The effect observed in clinical trials was replicated in routine practice as well. Seenan et al. [6] carried out a systematic review of 25 observational studies comparing death and other complications of stroke patients managed in SUs versus non-SU care. Results showed that patients experienced better survival (mortality odds ratio (OR) 0.79, 95\% confidence interval (CI) $0.73-0.86$ ) within 1 year of a stroke episode if they were treated in a SU.

In 2006, Canadian Best Practice Recommendations providing evidence-based practices for stroke management were released [7]. According to that document, a SU should consist of a geographically defined unit, dedicated multidisciplinary team with specialized expertise in stroke and have standardized processes of care and resources [7, 8]. This was followed by "A Guide to the Implementation of Stroke Unit Care", which focused on a set of considerations for the development and enhancement of SUs [9]. Nonetheless, implementation of SUs in Canada in comparison with other countries is low. According to the Canadian Stroke Network report (2011), only $23 \%$ of stroke patients were treated in SUs [10], compared with $>80 \%$ in Sweden (2006) [11], 75\% in England (2008), and 50\% in Australia (2009) [12].

Considering that implementation of SUs is a dynamic process, and that not all components of effective SUs are applicable or feasible for all facilities [9], it is important to identify the availability of SUs and the degree in which core components are currently in place. Determining the characteristics of SUs in Ontario, as well as their effectiveness and cost-effectiveness in a pragmatic setting, was the impetus for the project that was funded by the Ontario Stroke Network (OSN) and the Ontario Ministry of Health and Long-Term Care. The specific research objective of this study was to conduct a panprovincial survey of existing SUs in the province of Ontario to describe their availability, structure, the care they provide, and the population they serve.

\section{Methods}

The Ontario Stroke Unit survey was designed to collect data on characteristics of SUs. In order to identify all SUs in Ontario, we used the OSN database (2011) [13], followed by a snowball sampling technique. During snowball sampling, all respondents were asked whether they were aware of the existence of any other Ontario SUs [14].

A closed- and semi open-ended questionnaire, based on Canadian Best Practice Recommendations for Stroke Care [8] and Langhorne's findings on the effective components of SU care [15] (Additional file 1), was designed. Following an iterative process, the questionnaire was piloted, and once finalized, it was administered to the clinical and administrative leaders (managers, medical directors and team leaders) of the identified SUs. The questionnaire focused on: i) characteristics of SUs, such as structure and organization (geographic location, size and function of the unit); ii) staffing components (multidisciplinary team composition, their expertise, staffing levels and intensity); and iii) processes of care (patient population, diagnostic services, implementation of standardized protocols and valid assessment tools, rehabilitation and patient education).

An interviewer conducted a telephone survey with the SU managers in 2013-2014. The average survey duration was approximately $60 \mathrm{~min}$ per SU.

\section{Definition and regional distribution of SUs}

In this study we used the Canadian Stroke Strategy definition of SUs [9]:

I. Acute Stroke Unit (ASU): A specialized, geographically defined hospital unit dedicated to the management of stroke patients during the first seven to 10 days, or longer, following an acute stroke event and staffed by an interprofessional team.

II. Integrated Stroke Unit (ISU): A specialized, geographically defined hospital unit dedicated to the management of stroke patients. The unit provides both acute and rehabilitation care to patients during their inpatient stays following a stroke.

III.Rehabilitation Stroke Unit (RSU): A specialized, geographically defined rehabilitation unit dedicated to the rehabilitation of stroke patients.

Dedicated SU staff members were defined as someone who wase exclusively assigned to a SU. Dedicated beds 
were defined as beds that were exclusively allocated to stroke patients.

In Ontario there are 14 Local Health Integrational Networks (LHINs), which are responsible for centralized administration of health services within the province. We used boundaries of each LHIN to estimate geographic distribution of SUs [16]. The number of available SUs and stroke incident cases per LHIN (2012-2013) were used to approximate potential volume of stroke patients treated per SU [17].

\section{Statistical analyses}

Statistical analyses were conducted using SAS version 9.4 (SAS Institute Inc, Cary, North Carolina). Descriptive statistics were used to summarize survey responses. Continuous data were described using means/medians and standard deviations/interquartile range. Categorical data were summarized as frequency counts and percentages.

\section{Results}

Overall, 32 SUs were identified (21 ASUs, 10 ISUs and one RSU) (Additional file 2). Two of these were identified using the snowball sampling method. At least one respondent from each SU was interviewed (100\% response rate), with a total of 39 staff members interviewed across 32 SUs.

Surveys were conducted with program/unit managers $(n=13)$, clinic managers $(n=11)$, patient care managers $(n=4)$, or other administrative staff $(n=4)$. The additional staff members $(n=7)$ were aided in answering the questionnaire if required. SUs were available in all 14 LHINs except Central West (Fig. 1). The estimated average ratio of stroke patients served per SU was 604, with wide variability across the province [242 in the North East LHIN to 1480 in the Mississauga Halton LHIN (Table 1)]. An overwhelming majority $(n=24 ; 75 \%)$ of SUs were in operation for more than 5 years with an average duration of operation of $7.3 \pm 4.4$ years.

\section{Structure and organization}

The majority of SUs were located on general medicine wards $(n=18 ; 56 \%)$, followed by neurology or rehabilitation wards $(n=9 ; 28 \%)$. Few SUs defined themselves as a dedicated stand-alone SU with their physical location not being associated with a specific specialty ward $(n=5$;

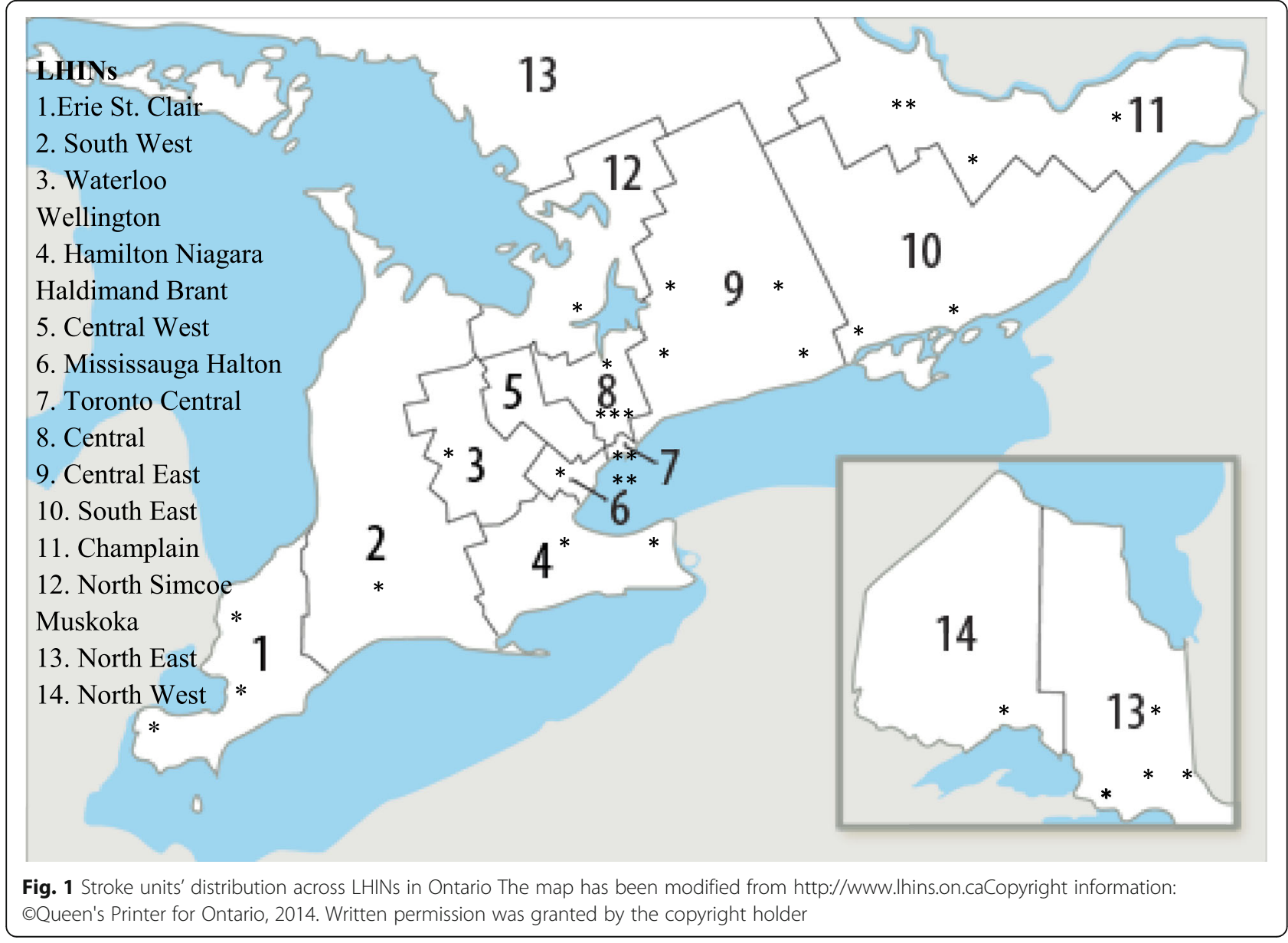


Table 1 Geographic distribution of stroke units and estimated access to care

\begin{tabular}{lllll}
\hline LHIN & \# of SU & $\begin{array}{l}\text { Total } \\
\text { population }\end{array}$ & $\begin{array}{l}\text { \# of stroke/ } \\
\text { TIA patients } \\
(2012-13)^{\mathrm{a}}\end{array}$ & $\begin{array}{l}\text { \# of stroke/ } \\
\text { TIA patients } \\
\text { per SU }\end{array}$ \\
\hline Erie St. Clair & 3 & 623,300 & 1,219 & 407 \\
South West & 1 & 890,100 & 1,376 & 1,376 \\
HNHB & 2 & $1,298,300$ & 2,544 & 1,272 \\
Waterloo Wellington & 1 & 679,700 & 996 & 996 \\
Mississauga Halton & 1 & $1,002,300$ & 1,480 & 1,480 \\
Central West & 0 & 735,200 & 805 & N/A \\
Central & 4 & $1,522,800$ & 1,734 & 434 \\
Central East & 4 & $1,419,800$ & 2,052 & 513 \\
Toronto Central & 4 & $1,075,100$ & 2,464 & 616 \\
North Simcoe Muskoka & 1 & 417,000 & 858 & 858 \\
South East & 2 & 457,200 & 861 & 431 \\
Champlain & 4 & $1,131,000$ & 1,821 & 456 \\
North East & 4 & 545,000 & 965 & 242 \\
North West & 1 & 231,900 & 452 & 452 \\
Ontario (total) & 32 & $12,028,900$ & 19,327 & 604
\end{tabular}

HNHB Hamilton Niagara Haldimand Brant, LHIN Local Health Integration Network, N/A Not Applicable, SU Stroke Unit, TIA Transient Ischemic Attack aData source: Ontario Stroke Evaluation Report 2014 [17]
16\%). Half of the ASUs had no beds exclusively allocated for stroke patients; but had priority beds. The median number of beds in ASUs and ISUs was 11 (range 4-37) and 19 (range 4-28), with an average of $80 \%$ and $91 \%$ being occupied on a typical day, respectively.

Although the reported average acute length of stay (LOS) did not vary significantly with the type of SU (ASU $=9.1 \pm 4.2$ days, $\mathrm{ISU}=8.2 \pm 2.9$ days), the full LOS was significantly longer in ISUs, due to rehabilitation services (the rehabilitation LOS was $21.0 \pm 10.9$ days). One fifth of SUs noted that waiting times for diagnostic services often increases LOS. Table 2 summarizes the structure and organization of SUs.

\section{SUs staff composition, workload and specialized training}

The majority of ASUs $(n=13,62 \%)$ and ISUs $(n=8$, $80 \%$ ) indicated that an internist was the most responsible physician for patient care (Table 2). One third of the SUs lacked a general neurologist or stroke neurologists on staff (ASU: $n=6 / 21$, ISU: $n=4 / 10$ ). A core multidisciplinary team (nursing, physiotherapist (PT), occupational therapist (OT), speech language pathologist (SLP), social worker (SW) and clinical nutritionists) was available in all SUs, either as dedicated staff or for consultation. There was no difference between ASUs and ISUs in dedicated allied health personnel median full time equivalent (FTE) (0.5-0.8 FTEs per 10 beds).

Table 2 Structure and organisation of SUs

\begin{tabular}{|c|c|c|c|c|}
\hline & Acute SUs $(n=21)$ & Integrated SUs $(n=10)$ & Rehabilitation SU $(n=1)$ & Total SUs $(n=32)$ \\
\hline Operation time (mean $\pm s d$, years) & $6.9 \pm 3.9$ & $8.6 \pm 5.2$ & $3 \pm 0$ & $7.3 \pm 4.4$ \\
\hline$<5$ years $(n, \%)$ & $7(33)$ & $2(20)$ & $1(100)$ & $10(31)$ \\
\hline$\geq 5$ years $(n, \%)$ & $14(67)$ & $8(80)$ & $0(0)$ & $22(69)$ \\
\hline \multicolumn{5}{|l|}{ Geographic location ( $n, \%)$} \\
\hline General medicine & $14(67)$ & $4(40)$ & $0(0)$ & $18(56)$ \\
\hline Neurology & $4(19)$ & $0(0)$ & $0(0)$ & $4(12)$ \\
\hline Rehabilitation & $0(0)$ & $4(40)$ & $1(100)$ & $5(16)$ \\
\hline Dedicated unit & $3(14)$ & $2(20)$ & $0(0)$ & $5(16)$ \\
\hline SUs with dedicated beds ( $n, \%)$ & $10(48)$ & $10(100)$ & $1(100)$ & $21(66)$ \\
\hline Number of beds (median, range) & $11(4-37)$ & $19(4-28)$ & $6(-)$ & $13(4-37)$ \\
\hline Mean bed occupancy percentage on a usual day (range) & $80 \%(50-100)$ & $91 \%(60-100)$ & $83 \%(-)$ & $84 \%(50-100)$ \\
\hline Acute LOS (mean $\pm s d$, days) & $9.1 \pm 4.2$ & $8.2 \pm 2.9$ & - & $8.8 \pm 3.7$ \\
\hline Rehabilitation LOS (mean \pm sd, days) & - & $21.0 \pm 10.9$ & $38 \pm 0$ & $23.6 \pm 10.7$ \\
\hline \multicolumn{5}{|l|}{ Responsible physician for patient care } \\
\hline Stroke Neurologist (n, \%) & $6(29)$ & $1(10)$ & $0(0)$ & $7(22)$ \\
\hline General neurologist ( $n, \%)$ & $7(33)$ & $1(10)$ & $0(0)$ & $8(25)$ \\
\hline Internist ( $n, \%)$ & $13(62)$ & $8(80)$ & $0(0)$ & $21(66)$ \\
\hline Family physician ( $n, \%)$ & $3(14)$ & $5(50)$ & $1(100)$ & $9(28)$ \\
\hline
\end{tabular}

Data was calculated after excluding missing values LOS Length of Stay, SUs Stroke Units, SD Standard Deviation ${ }^{a}$ Beds that are exclusively allocated for stroke patients 
However, ASUs had substantially higher FTEs for nurses, educators and care managers (Table 3). Data regarding FTEs for non-dedicated staff was not collected.

Table 3 Composition and staffing details for multidisciplinary team in ASUs and ISUs

\begin{tabular}{llll}
\hline $\begin{array}{l}\text { Dedicated } \\
\mathrm{n}(\%)\end{array}$ & $\begin{array}{l}\text { Consultation } \\
\mathrm{n}(\%)\end{array}$ & $\begin{array}{l}\text { FTE per 10 } \\
\text { bed }^{\mathrm{a}} \text { median } \\
\text { (range) }\end{array}$ & $\begin{array}{l}\text { Patient to } \\
\text { staff ratio }\end{array}$ \\
\hline
\end{tabular}

Acute SUs $(n=21)$

\begin{tabular}{|c|c|c|c|c|}
\hline PT & $10(47.6)$ & $11(52.4)$ & $0.8(0.6-2.7)$ & $12: 1$ \\
\hline OT & $10(47.6)$ & $11(52.4)$ & $0.8(0.5-2.0)$ & 12:1 \\
\hline SLP & $6(28.6)$ & $15(71.4)$ & $0.6(0.3-1.8)$ & 12:1 \\
\hline SW & $7(33.3)$ & $14(66.7)$ & $0.5(0.3-1.0)$ & - \\
\hline Nutritionist & $5(23.8)$ & $16(76.1)$ & $0.2(0.2-1.0)$ & - \\
\hline Pharmacist & $5(23.8)$ & $16(76.1)$ & $1.0(1.0-2.0)$ & - \\
\hline Psychologist & - & $7(33.3)$ & - & - \\
\hline RN & $6(28.6)$ & $15(71.4)$ & $11.3(1.0-17.0)$ & \\
\hline Day-shift & & & & $4: 1$ \\
\hline Night-shift & & & & $6: 1$ \\
\hline RPN & $4(19.1)$ & $13(61.9)$ & $11.0(2.9-12.5)$ & \\
\hline Day-shift & & & & $5: 1$ \\
\hline Night-shift & & & & $7: 1$ \\
\hline APN & $5(23.8)$ & $3(14.3)$ & $0.8(0.3-1.0)$ & - \\
\hline Educator & $6(28.6)$ & $15(71.4)$ & $0.4(0.2-1.7)$ & - \\
\hline Admin. staff & $5(23.8)$ & $11(52.4)$ & $0.6(0.1-1.3)$ & - \\
\hline Care manager & $6(28.6)$ & $12(57.1)$ & $1.0(0.4-2.5)$ & - \\
\hline \multicolumn{5}{|c|}{ tegrated SUs $(n=10)$} \\
\hline$P$ & $6(66.7)$ & $3(33.3)$ & $0.8(0.5-1.0)$ & 12:1 \\
\hline OT & $6(66.7)$ & $3(33.3)$ & $0.8(0.5-1.0)$ & $13: 1$ \\
\hline SLP & $5(55.6)$ & $4(44.4)$ & $0.5(0.3-0.6)$ & $14: 1$ \\
\hline SW & $5(55.6)$ & $4(44.4)$ & $0.5(0.2-4.0)$ & - \\
\hline Nutritionist & $4(44.4)$ & $5(55.6)$ & $0.5(0.2-0.6)$ & - \\
\hline Pharmacist & $3(33.3)$ & $6(66.7)$ & $0.8(0.5-1.0)$ & - \\
\hline Psychologist & - & $3(33.3)$ & - & - \\
\hline RN & $8(88.9)$ & $1(11.1)$ & $8.0(3.5-13.0)$ & \\
\hline Day-shift & & & & $4: 1$ \\
\hline Night-shift & & & & $7: 1$ \\
\hline RPN & $7(77.8)$ & $1(11.1)$ & $4.2(1.9-15.0)$ & \\
\hline Day-shift & & & & $5: 1$ \\
\hline Night-shift & & & & $7: 1$ \\
\hline APN & $2(22.2)$ & $3(33.3)$ & $0.3(0.2-0.4)$ & - \\
\hline Educator & $2(22.2)$ & $7(77.8)$ & $0.9(0.2-1.7)$ & - \\
\hline Admin. staff & $7(77.8)$ & $1(11.1)$ & $1.0(0.4-1.8)$ & - \\
\hline Care manager & $6(66.6)$ & $3(33.3)$ & $0.4(0.2-0.8)$ & - \\
\hline
\end{tabular}

Data was calculated after excluding missing values APN Advanced Practice Nurse, FTE Full Time Equivalent, OT Occupational Therapist, $P T$ Physiotherapist, $R N$ Registered Nurse, RPN Registered Practical Nurse, SLP Speech Language Pathologist, SW Social Worker, SUs Stroke Units ${ }^{\mathrm{a}} \mathrm{FTE}$ presented for dedicated personnel only
Both acute and integrated SUs had nurse-to-patient ratios of 1:4-5 during the day and 1:6-7 during the night with no difference between weekdays and weekends. There was little difference in the allied health staff-topatient ratios; ASUs $=1: 12$ for PT, OT and SLP and in ISU there was 1:12 for PT; 1:13 for OT and 1:14 for SLP (Table 3).

Staff educational sessions that included both conferences and in-house talks in the SUs were attended on a monthly and yearly basis. One third of SUs $(n=10 ; 31 \%)$ indicated having budget allocated for training purposes, and only two thirds of the ISUs $(n=6)$ had nursing staff with specialized training in stroke care.

\section{SUs patient population and processes of care}

Typically, patients were admitted with a clinical diagnosis of either ischemic or hemorrhagic stroke. ASUs were more likely to admit patients with transitory ischemic attack (TIA) compared with ISUs $(n=17,80 \%$ vs $n=6$, $60 \%)$. The majority of SUs $(n=18 ; 56.3 \%)$ did not admit unconscious patients (Fig. 2). Some SUs excluded patients with: symptoms lasting more than one week and severe co-morbidities, subdural hemorrhage and subarachnoid hemorrhage, patients with only transient symptoms, or prior dependency (Fig. 2).

Almost all SUs had access to computed tomography (CT) $(n=31,97 \%)$, magnetic resonance imaging (MRI) $(n=30,97 \%)$ and carotid Doppler ultrasound $(n=30$, $97 \%)$. The access to on site surgical interventions was limited: neuro/-vascular surgery $(n=12,37.5 \%)$, interventional neurology $(n=13,41 \%)$ and neuroradiology $(n=16,50 \%)$. The administration of different valid assessment scales is summarized in Table 4 . The vast majority of SUs had written protocols $(n=22 ; 69 \%)$ and standardized order sets $(n=32 ; 100 \%)$ in place to evaluate stroke-related impairments and further guide stroke care. Monitoring of skin surface areas for pressure ulcer prevention was reported in all SUs (Table 4).

Early discharge assessment (within $48 \mathrm{~h}$ ) was conducted in nearly two thirds of SUs $(n=20 ; 62 \%)$. Rehabilitation services were being provided on site in ISUs. Formal interdisciplinary team meetings were being conducted at minimum once a week in all but two SUs. During the meetings a goal setting $(n=29,91 \%)$ and individualized rehabilitation care plans $(n=23,72 \%)$ were discussed by majority of the units.

All SUs routinely provided educational material to patients and caregivers with information on stroke and rehabilitation, risk management, and availability of community resources. The most common formats of education were one-on-one teaching modules and pamphlets.

All core elements of SU care (geographically defined unit, multidisciplinary specialized staff, in-house diagnostic services, administration of valid assessment tools 


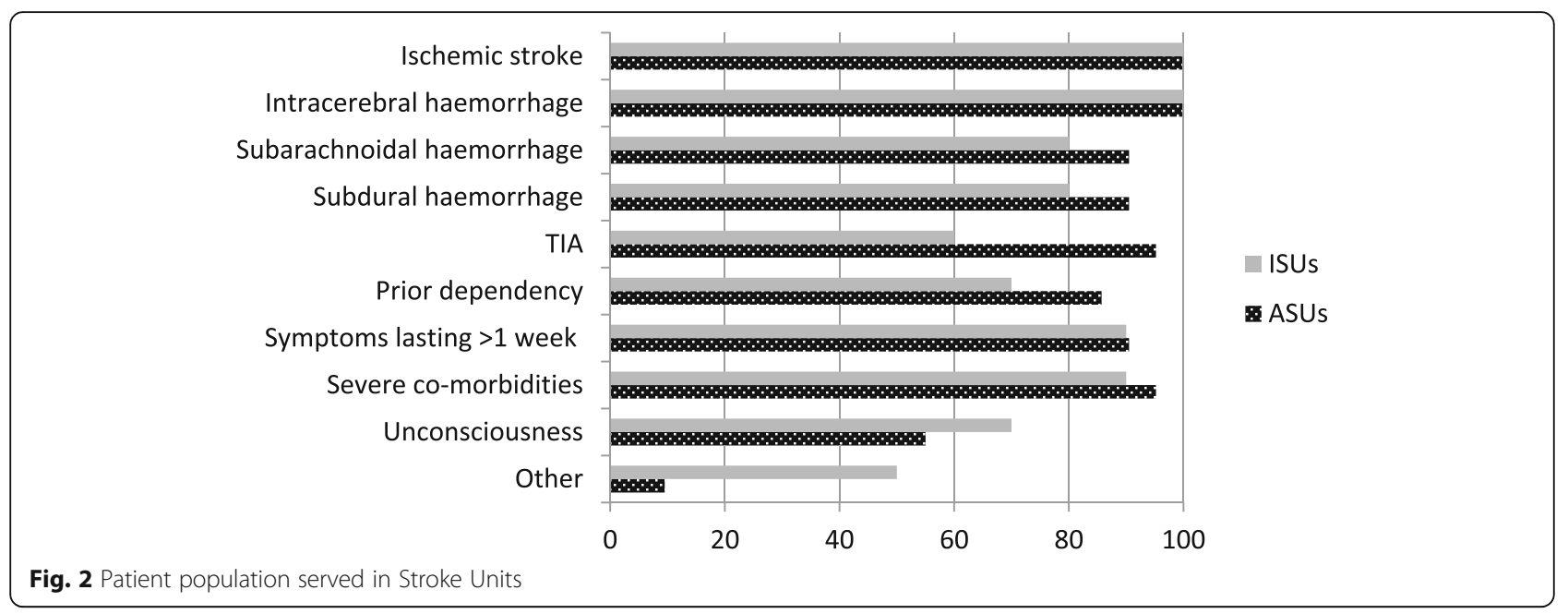

and implementation of standardized protocols, team meetings, rehabilitation and patient education) were addressed by 16 out of 32 units (50\%) (Table 5). There was no noticeable clustering of deficient core elements in certain SUs, or in particular geographic regions (i.e. by LHIN). The main areas for improvement included specialized nurse training in stroke care (met by 26 units), provision of individualized rehabilitation care plans (met by 23 units), and early discharge assessment (met by 20 units) conducted within $48 \mathrm{~h}$ of admission.

\section{Discussion}

We conducted a comprehensive survey of 32 SUs in Ontario, which included 21 ASUs, 10 ISUs and one RSU. According to the Canadian Best Practice Recommendations for Stroke Care, SUs should consist of a geographically defined unit with a dedicated multidisciplinary team with specialized expertise in stroke and standardized processes of care and resources [8]. The study revealed regional variation in access to SUs. We also found that many of the core components of SUs are in place, however half of the SUs in Ontario do not meet all criteria.

\section{SU regional distribution, structure and organization}

This study demonstrated that the SUs distribution varied between regions, with no SU available in the Central West LHIN. There was a greater than six-fold difference in stroke/TIA population served by the SUs, ranging from 242 in the North East LHIN to 1480 in the Mississauga Halton LHIN. Potential disparity in access to SU care might be supported by the fact that only $19 \%$ (27 out of 142) of acute care hospitals in Ontario had a SU in 20102011, and only $38.3 \%$ of admitted stroke patients were treated in SUs [18]. The access was dramatically lower compared with European countries, (2010-2011) where over $60 \%$ of stroke patients received care in SUs $[19,20]$.
Evidence from clinical trials and observational studies suggests that stroke care provided in geographically defined SUs is associated with a $20 \%$ lower chance of death relative to care in general wards $[4,6]$. In the current study, all 32 identified SUs had a geographically defined location, and the majority were based in a general medicine ward with a median of 13 beds. At the time of the survey, Canadian recommendations did not require SUs to be exclusive to stroke patients, nor have all of the resources in place; however, recommendations did require SUs to be in one geographic location [17]. Later in 2014, the OSN revised the definition of SUs, which included additional criteria related to bed allocation and staff. According to the new definition "a stroke unit is a geographical unit with identifiable co-located beds that are occupied by stroke patients $75 \%$ of the time and have a dedicated interprofessional team with expertise in stroke care including, at a minimum, nursing, physiotherapy, occupational therapy and speech-language pathology" [17]. Though our questionnaire did not specifically ask for the number of beds occupied exclusively by stroke patients, we can speculate that the ASUs with no dedicated beds for stroke patients (half of ASUs) might fail to meet that criterion. This could partially explain decreased number of SUs $(n=14)$ identified in 2014 by OSN that satisfied the new definition [17]. European guidelines are more restrictive and require $\mathrm{SUs}$ to be exclusive for stroke and TIA patients only, and not to admit patients with other disorders [21]. The goal is to train staff to better address the needs of stroke patients and gain specialized skills and expertise, which could translate into improved patient outcomes [21]. In the observational study looking into mortality for 26000 stroke patients, Saposnik et al., concluded that seven day mortality was 9.5\% for hospitals with a volume of less than 50 stroke patients per year, $7.3 \%$ for a volume of 100-199 per year, and $6.0 \%$ for a volume of more than $200(<0.001)$ [22]. 
Table 4 Processes of care in Stroke Units

\begin{tabular}{|c|c|c|c|}
\hline$n(\%)$ & $\begin{array}{l}\text { Acute SUs } \\
(n=21)\end{array}$ & $\begin{array}{l}\text { Integrated } \\
\text { SUs }(n=10)\end{array}$ & $\begin{array}{l}\text { Total } \\
(n=32)^{\mathrm{a}}\end{array}$ \\
\hline \multicolumn{4}{|l|}{ Available diagnostic procedures } \\
\hline$C T$ & $21(100.0)$ & $10(100.0)$ & $31(96.9)$ \\
\hline$M R I$ & $21(100.0)$ & $9(90.0)$ & $30(93.8)$ \\
\hline Cerebral angiography & $18(85.7)$ & $8(88.0)$ & $26(83.8)$ \\
\hline Carotid Doppler ultrasound & $20(95.2)$ & $10(100.0)$ & $30(93.8)$ \\
\hline \multicolumn{4}{|l|}{ Available on-site surgical therapy } \\
\hline Neuro/-vascular surgery & $9(42.9)$ & $3(33.3)$ & $12(37.5)$ \\
\hline Interventional neurology & $10(47.6)$ & $3(33.3)$ & $13(40.6)$ \\
\hline Interventional neuroradiology & $12(57.1)$ & $4(44.4)$ & $16(50.0)$ \\
\hline \multicolumn{4}{|c|}{ Standardized valid assessments scales } \\
\hline Barthel index & $8(38.1)$ & $6(60.0)$ & $14(43.7)$ \\
\hline Canadian neurological scale & $18(85.7)$ & $7(70.0)$ & $25(78.1)$ \\
\hline NIHSS & $14(66.7)$ & $7(70.0)$ & $21(65.6)$ \\
\hline FIM & $20(95.2)$ & $10(100.0)$ & $30(93.8)$ \\
\hline TORBSST & $10(47.6)$ & $5(50.0)$ & $15(46.8)$ \\
\hline Braden scale & $20(95.2)$ & $10(100.0)$ & $31(96.8)$ \\
\hline \multicolumn{4}{|l|}{ Approaches to guide stroke care } \\
\hline Written protocols & $13(65.0)$ & $8(80.0)$ & $22(70.9)$ \\
\hline Standardized order sets & $21(100.0)$ & $10(100.0)$ & $32(100.0)$ \\
\hline Monitoring of pressure area & $21(100.0)$ & $10(100.0)$ & $32(100.0)$ \\
\hline $\begin{array}{l}\text { Interdisciplinary team } \\
\text { meeting } \geq 1 / \text { week }\end{array}$ & $20(95.2)$ & $10(100.0)$ & $30(93.8)$ \\
\hline Goal setting & $19(90.5)$ & $9(90.0)$ & $29(90.6)$ \\
\hline $\begin{array}{l}\text { Creation of individualized } \\
\text { care plans }\end{array}$ & $12(57.1)$ & $10(100)$ & 23(71.8) \\
\hline \multicolumn{4}{|c|}{ Timing of initial discharge assessment } \\
\hline$<24 h$ & $7(33.3)$ & $2(20.0)$ & $9(28.3)$ \\
\hline $1-2 d$ & $6(28.6)$ & $5(50.0)$ & $11(34.4)$ \\
\hline $3-4 d$ & $5(23.8)$ & $3(30.0)$ & $8(25.0)$ \\
\hline$<4 d$ & $3(14.3)$ & $0(0.0)$ & $3(12.5)$ \\
\hline
\end{tabular}

CT Computed Tomography, FIM Functional Independence Measure, MRI Magnetic Resonance Imaging, NIHSS National Institute Health Stroke Scale, TORBSST Toronto Bedside Swallowing Screening Test, SUs Stroke Units

${ }^{\text {a}}$ Total SUs column includes information on the Rehabilitation unit $(n=1)$ as well

\section{SUs staff composition, workload and specialized training}

Nursing and allied health staff was available in all units, either as dedicated staff or on a consultation basis. However, there still might be room for improvement, such as adjustment in staffing levels and an optimization in the staff-to-patient ratio. FTE for allied health professionals ( 0.5-0.8 FTE per type per 10 beds) was somewhat lower than reported in European studies (PT/OT 1-2, for SLP 0.2-0.6 [23, 24]). This is also supported by our finding of the high patient-to-therapist ratio (12-14:1), which was almost double than reported in US clinics (5-
Table $\mathbf{5}$ Summary of core elements met by SUs

\begin{tabular}{ll}
\hline Core elements for SUs & SU, n (\%) \\
\hline Geographically defined unit & $32(100.0)$ \\
Multidisciplinary team (physician, nurse, & $32(100.0)$ \\
OT/PT/SLP/clinical nutritionist) & \\
Advance training in stroke management ${ }^{\mathrm{a}}$ & $26(81.3)$ \\
Acute imaging (MRI/CT) & $32(100.0)$ \\
Valid scales (CNS/NIHSS/FIM) & $30(93.8)$ \\
Standardized order sets/protocols/algorithms & $32(100.0)$ \\
Interdisciplinary team meeting $\geq 1$ per week & $31(96.9)$ \\
Creation of individualized rehabilitation care plans & $23(71.8)$ \\
Goal setting & $29(90.6)$ \\
Early discharge assessment (<48 h of admission) & $20(62.5)$ \\
Patient/carer education & $32(100.0)$ \\
In place rehabilitation (for Integrated SUs only) & $10(100.0)$ \\
All core elements met & $16(50.0)$ \\
\hline
\end{tabular}

Core elements were adopted from the Canadian Best

Practices Recommendation

CNS Canadian Neurological Scale, CT Computed Tomography, FIM Functional Independence Measure, MRI Magnetic Resonance Imaging, NIHSS National Institute Health Stroke Scale, OT Occupational Therapist, PT Physiotherapist, SLP Speech Language Pathologist, SUs Stroke Units

${ }^{a}$ Data on nurses training only

8 :1) [25]. Nursing allocation (8-10 FTE per 10 beds) was comparable to other studies. Across 92 surveyed SUs in England, a median of 11 nurses per 10 beds was recorded [23]. Recommendations regarding nurse staffing levels vary across countries and depend on local contexts and delivery models. Scottish Guidelines recommend having 10 nurses [24], while European Stroke Organization requires minimum of 15 nurses per 10 monitored beds [21]. Using data from Canadian practice and several other studies, Phillips et al., derived the following FTE allocation (per 10 beds) for multidisciplinary staff: RN -14; OT/PT/SLP - 1.0 each; and SW - 0.5 [26]. In our study only a few SUs reported on availability of an advanced practice nurse (APN) $(n=5,16 \%)$, which according to the Brain Attack Coalition is a vital member of a stroke team who provides support with patient care, educational programs, research activities and quality assurance [27].

Stroke-focused training for nurses might be considered as another major area of improvement, since only two thirds of the ISUs included nurses who received specialized training in stroke care. International stroke experts consider nurses trained in stroke management as an absolutely integral element of care [19]. The German Stroke Society has established a certified stroke nursing curriculum with over $200 \mathrm{~h}$ of training [19]. Lack of specialized nurse training in Ontario might be explained by scarce resources, since only one third of ISUs had specific funds allocated for training purposes. Continuing stroke education is important not only for nurses, but 
also for other medical staff, since internists were the most frequently reported responsible physicians for patient care. Our questionnaire did not capture information on physician training in stroke management; however general neurologists or stroke neurologists were more likely to receive such training, rather than internists. In Ontario, it was found that one third of the SUs lacked a general neurologist or stroke neurologists on staff. In contrast, 196 out of 200 SUs in Germany were run by neurologists and only four by an internist [19]. In a survey conducted by the US Brain Attack Coalition, leaders and experts in cerebrovascular disease rated having a stroke neurologist on staff as an important element of a stroke centre [27]. Furthermore, in a nationwide prospective observational study, Goldstein et al. revealed that patients with ischemic stroke who were treated by neurologists experienced improved outcomes, such as less dependency or mortality $(\mathrm{OR}=0.64 ; 95 \% \mathrm{CI} 0.45$ to 0.92) than patients who were cared for by nonneurologists [28].

\section{Patient population and processes of care}

In addition to ischemic and haemorrhagic stroke patients, most of the acute and integrated SUs also admitted patients with transient symptoms, prior dependency, and severe co-morbidities. Recommendations indicate that all patients with a suspected diagnosis of stroke urgently undergo brain imaging (CTs or MRIs) [8, 21, 27]. Although specific diagnostic imaging necessary for stroke patient management was highly available in SUs, one fifth of SUs mentioned that waiting times for diagnostic services often increase LOS. Although our study questionnaire did not specifically address acute imaging wait times,, only $22 \%$ of stroke patients received a scan within one hour of arrival according to the Canadian Stroke Network 2011 report [10]. This emphasizes the need for written protocols and standardized order sets that will prioritize the access of stroke patient to diagnostic services and time-sensitive treatments. Phillips et al. acknowledged that having standardized order sets in their SU in Halifax not only improved recording of the information and its accessibility to the stroke team, but also facilitated interdisciplinary assessment and treatment [29]. Although all SUs mentioned the availability of standardized order sets, only two thirds of SUs had written protocols in place to guide stroke care.

It was encouraging to report the administration of Braden Scale for pressure ulcer monitoring as a part of daily routine in all SUs. This may play an important role in pressure ulcer prevention [30], as pressure ulcers increase health services utilization and impair quality of life [31]. The administration of validated scales to monitor mental and motor functions of stroke patients (Canadian Neurological Scale, the National Institutes of
Health Stroke Scale and Functional Independence Measure [32-34]) was high in SUs $(66 \%-100 \%)$, and was comparable to other surveys conducted by Langhorne et al. [15].

Early discharge services and rehabilitation are cornerstones of stroke patient management. In the metaanalysis of 11 trials from six countries (Australia, Canada, Norway, Sweden, Thailand, UK) Langhorne et al., showed that early supported discharge reduced the risk of death or long-term dependency (OR 0.79 95\% CI 0.64 to 0.97 ) in stroke patients, as well as shortened LOS [35]. Meanwhile, Kwakkel et al. reported positive association between intensity of rehabilitation therapies and functional recovery following stroke [36]. Though our questionnaire was not designed to capture patient level data, it seems that rehabilitation services need to be improved, since not all units conducted early discharge assessment $(n=20,63 \%)$ or provided individualized rehabilitation care plans $(n=23,72 \%)$.

A Cochrane review by Smith et al. assessed the effectiveness of strategies for information provision to stroke patients and their caregivers. They supported routine provision of information; however, there was no preferred method of communication [37]. In this study, SU managers mentioned that the most common format of patient education were pamphlets and one-to-one teaching modules.

Based on our survey results the following areas should be prioritized to improve stroke care in Ontario: i) optimization of staffing level and intensity for nurses and allied health professionals, ii) addressing the lack of general neurologists and stroke neurologists in SUs iii) specialized stroke care training for a multidisciplinary team, iv) priority bed allocation for stroke patients, v) provision of individualized rehabilitation plans and early discharge assessment.

\section{Strength and limitations}

Employing two sampling strategies for this panprovincial scan, we were able to identify two additional SUs in addition to those listed by the OSN in 2011 [13]. With our 100\% response rate we were able capture core characteristics, organisational structures and care processes of the 32 identified SUs. The first limitation of the study was that it was based on feedback from SU managers and consequently, interpretations of staff-topatient ratios should be made with caution. In our study, dedicated staffing levels and full-time equivalent (FTE) information for stroke teams were difficult to obtain, since different levels of cross-cover with other nonstroke services (e.g. general ward, neurology) were reported. In addition, staffing ratios may not be easily comparable to other jurisdictions, since studies defined and calculated staffing levels in various ways. 
Secondly, there was potential underreporting of some procedures; i.e. managers of SUs might had been unaware of the administration of specific diagnostic tools for an impairment assessment). The survey questionnaire did not cover all important components of stroke care, i.e. assessment of treatment package, early mobilisation practices, and complication management.

Finally, as noted above, the survey was conducted before the OSN revised the definition of a SU in 2014, which introduced new criteria on co-allocated beds and its occupancy rate by stroke patients [17]. This led to a reduction in the number of available SUs in Ontario to 14, which all satisfied the new criterion.

\section{Conclusion}

This study illustrated the heterogeneous structure of SUs in Ontario and signalled potential disparity in access to SU care. Only half of the identified SUs met all core criteria set forth by the Canadian Stroke Strategy Guide to the Implementation of Stroke Unit Care 2009 [9]. Finally, this study has revealed important areas for improvement in SU care, such as stroke care training for a multidisciplinary team, adjustment in staffing level and intensity for nurses and allied health professionals, bed allocation for stroke patients, and the provisioning of individualized rehabilitation plans and early discharge assessment.

\section{Additional files}

Additional file 1: Organized Inpatient (Stroke Unit) Care Questionnaire Blank version of the survey questionnaire. (DOCX 24 kb)

Additional file 2: Distribution of identified Stroke Units across Local Health Integrational Networks. This file lists all identified and surveyed stroke units across Local Health Integrational Networks. (DOCX 16 kb)

\section{Abbreviations \\ ASU: Acute stroke unit; Cl: Confidence interval; CT: Computed tomography; FTE: Full time equivalent; ISU: Integrated stroke unit; LHIN: Local health integrational network; LOS: Length of stay; MRI: Magnetic resonance imaging; OR: Odds ratio; OSN: Ontario stroke network; OT: Occupational therapist; PT: Physiotherapist; RSU: Rehabilitation stroke unit; SLP: Speech language pathologist; SU: Stroke unit; SW: Social worker; TIA: Transitory ischemic attack; UK: United Kingdom; US: United States}

\section{Acknowledgments}

The authors gratefully acknowledge all participating stroke units, Ontario Stroke Network, and Ontario Ministry of health and Long-Term Care for collaboration and support. We also would like to thank Carcone S. for editing the manuscript.

\section{Funding}

This project was funded by a grant from the Ontario Ministry of Health and Long-Term Care, administered and supported by the Ontario Stroke Network The views expressed do not necessarily reflect those of the Ministry or the Ontario Stroke Network.

Moira Kapral was supported by a Career Investigator Award from the Hear and Stroke Foundation of Canada, Ontario Provincial Office. Murray Krahn was supported by the F. Norman Hughes Chair in Pharmacoeconomics, Leslie Dan Faculty of Pharmacy.

\section{Availability of data and materials}

The datasets used and/or analysed during the current study are available from the corresponding author on reasonable request.

\section{Authors' contributions}

VER, MK - conceived, designed and oversaw implementation of the study, participated in interpretation of study data, and critically revised the manuscript for important intellectual content. YS led analysis, interpretation of the data, and drafted the manuscript. IF made substantial contribution in study implementation and data acquisition. LI, GV participated in design, implementation of the study and critical revision of manuscript. MKK, MB, $\mathrm{RH}$, LK contributed to study design, participated in interpretation of study data, and critically revision of manuscript for important intellectual content. All authors read and approved the final manuscript.

\section{Competing interests}

The authors declare that they have no competing interests.

Consent for publication

Not applicable.

\section{Ethics approval and consent to participate}

The ethics review board at the University of Toronto approved the study protocol. Separate institutional review board approvals were obtained at each participating SU. Consent to participate in the survey was received from all interviewed participants of each identified SU.

\section{Author details}

${ }^{1}$ Toronto Health Economics and Technology Assessment (THETA) Collaborative, Toronto General Research Institute, Toronto General Hospital, University Health Network, University of Toronto, Toronto, ON, Canada. ${ }^{2}$ Institute of Health Policy, Management and Evaluation (IHPME), University of Toronto, Toronto, ON, Canada. ${ }^{3}$ Cancer Care Ontario, Toronto, ON, Canada. ${ }^{4}$ Institute for Clinical Evaluative Sciences (ICES), Toronto, ON, Canada. ${ }^{5}$ Ontario Stroke Network, Toronto, ON, Canada. ${ }^{6}$ Leslie Dan Faculty of Pharmacy, University of Toronto, Toronto, ON, Canada. ${ }^{7}$ Toronto General Hospital, University Health Network, University of Toronto, Toronto, ON, Canada. ${ }^{8}$ Faculty of Medicine, University of Toronto, Toronto, ON, Canada.

${ }^{9}$ Toronto Rehab Foundations, University Health Network, Toronto, ON, Canada.

Received: 20 April 2016 Accepted: 15 February 2017

Published online: 21 February 2017

\section{References}

1. World Heart Federation. Stroke. http://www.world-heart-federation.org/ cardiovascular-health/stroke/. Accessed 20 Dec 2015

2. Di Carlo A. Human and economic burden of stroke. Age Ageing. 2009;38(1): 4-5.

3. Mackay J, Mensah G. The Atlas of Heart Disease and Stroke. Geneva, Switzerland: World Health Organization; 2004. 2016 (March 24).

4. Stroke Unit Trialists' Collaboration. Organised inpatient (stroke unit) care for stroke. Cochrane Database Syst Rev. 2007:4:CD000197.

5. Stroke Unit Trialists' Collaboration. Organised inpatient (stroke unit) care for stroke. Cochrane Database Syst Rev. 2013;9:CD000197.

6. Seenan $P$, Long $M$, Langhorne P. Stroke units in their natural habitat: systematic review of observational studies. Stroke. 2007;38(6):1886-92.

7. The Canadian Stroke Strategy. Canadian Best Practice Recommendations for Stroke Care: 2006. Ottawa. http://www.strokecenter.org/wp-content/ uploads/2011/08/CSSManualENG_WEB_Sept07.pdf. Accessed 19 Mar 2016

8. Lindsay M, Gubitz G, Bayley M, Hill M, Davies-Schinkel C, Singh S, Canadian Best Practice Recommendations for Stroke Care (Update 2010). On behalf of the Canadian Stroke Strategy Best Practices and Standards Writing Group Ottawa. Ontario Canada: Canadian Stroke Network; 2010

9. Canadian Stroke Network \& Heart and Stroke Foundation. A Guide to the Implementation of Stroke Unit Care. Canadian Stroke Strategy. 2009. http:// strokebestpractices.ca/wp-content/uploads/2010/11/CSS-Stroke-UnitResource_EN-Final2-for-print.pdf. Accessed 18 Feb 2017.

10. Canadian Stroke Network. The quality of Stroke care in Canada. 2011.

11. Kaste M, Boysen $G$, Indredavik B, Norrving B. Stroke unit care in Scandinavian countries. Int J Stroke. 2006;1(1):44 
12. Cote, B. Tessier, A. Organisation of Stroke Care Services: Review of the Evidence, Policies and Experiences. Summary. ETMIS 2011. https://www. inesss.qc.ca/fileadmin/doc/INESSS/Rapports/OrganisationsSoins/INESSS_ Summary_AVC1_EN.pdf. Accessed 4 Mar 2016

13. Hall R, Khan F, O'Callaghan C, Meyer S, Fang J, Hodwitz K, Bayley M. Ontario Stroke Evaluation Report 2011: Improving System Efficiency by Implementing Stroke Best Practices. Toronto: Institute for Clinical Evaluative Sciences; 2011.

14. Heckathorn D. Respondent-driven sampling: A new approach to the study of hidden populations. Soc Probl. 1997;44:174-99.

15. Langhorne P, Pollock A. in conjunction with the Stroke Unit Trialists' Collaboration. What are the components of effective stroke unit care? Age Ageing. 2002;31:365-71.

16. Wijeysundera HC, Trubiani G, Abrahamyan L, Mitsakakis N, Witteman W, Paulden M, van der Velde G, Kingsbury K, Krahn M. Specialized multidisciplinary heart failure clinics in Ontario, Canada: an environmental scan. BMC Health Serv Res. 2012;12:236.

17. Hall R, Khan F, O'Callaghan C, Kapral M, Cullen A, Levi J, Wu J, Fang J, Bayley M. Ontario Stroke Evaluation Report 2014: On Target for Stroke Prevention and Care. Toronto: Institute for Clinical Evaluative Sciences; 2014.

18. Hall R, Khan F, O'Callaghan C, Kapral M, Hodwitz K, Fang J, Bayley M. Ontario stroke evaluation report: Prescribing system solutions to improve stroke outcomes: Ontario Stroke Report Card, 2010/11. Toronto: Institute for Clinical Evaluative Sciences; 2012.

19. Ringelstein E, Busse O, Ritter AM. Current concepts of Stroke Units in Germany and Europe. Schweiz Arch Neurol Psychiatr. 2011;162(4):155-60.

20. National Health Services Scotland. Scottish Stroke Care Audit, 2011 National Report: Stroke Services in Scottish Hospitals. Edinburgh 2011. http://www. strokeaudit.scot.nhs.uk/Downloads/2011_Report/SSCA-report-2011-webversion_new.pdf. Accessed 14 Mar 2016

21. Ringelstein E, Chamorro A, Kaste M, Langhorne P, Leys D, Lyrer P, Thijs V, Thomassen L, Toni D, for the ESO Stroke Unit Certification Committee. European stroke organisation recommendations to establish a stroke unit and stroke center. Stroke. 2013;44:828-40.

22. Saposnik G, Baibergenova A, O'Donnell M, Hill MD, Kapral MK, Hachinski V, Stroke Outcome Research Canada Working G. Hospital volume and stroke outcome: does it matter? Neurology. 2007;69(11):1142-51.

23. Rudd A, Jenkinson D, Grant R, Hoffman A. Staffing levels and patient dependence in English stroke units. Clin Med. 2009;9(2):110-5.

24. Scottish Intercollegiate Guidelines Network. Management of Patients with Stroke: Rehabilitation, Prevention and Management of Complications, and Discharge Planning, vol. 118. Edinburgh, Scotland: SIGN Publication; 2010. p. 108.

25. Conroy BE, DeJong G, Horn SD. Hospital-based stroke rehabilitation in the United States. Top Stroke Rehabil. 2009;16(1):34-43.

26. Phillips S, Corning C, Gill N, Edwards L. Stroke unit sizing and staffing: stroke system planning in Nova Scotia, Canada. Abstract. Int J Stroke. 2008;3 Suppl 1:306.

27. Alberts MJ, Latchaw RE, Selman WR, Shephard T, Hadley MN, Brass LM, Koroshetz W, Marler JR, Booss J, Zorowitz RD, et al. Recommendations for comprehensive stroke centers: a consensus statement from the Brain Attack Coalition. Stroke. 2005;36(7):1597-616.

28. Goldstein LB, Matchar DB, Hoff-Lindquist J, Samsa GP, Horner RD. VA Stroke Study: neurologist care is associated with increased testing but improved outcomes. Neurology. 2003:61(6):792-6.

29. Phillips SJ, Eskes GA, Gubitz GJ. Description and evaluation of an acute stroke unit. CMAJ. 2002;167(6):655-60.

30. Summers D, Leonard A, Wentworth D, Saver J, Simpson J, Spilker J, Hock N, Miller $E$, Mitchell $P$, on behalf of the American Heart Association Council on Cardiovascular Nursing and the Stroke Council. Comprehensive overview of nursing and interdisciplinary care of the acute ischemic stroke patient: a scientific statement from the American Heart Association. Stroke. 2009;40: 2911-44.

31. Thein HH, Gomes T, Krahn MD, Wodchis WP. Health status utilities and the impact of pressure ulcers in long-term care residents in Ontario. Qual Life Res. 2010;19(1):81-9.

32. Côté R, Battista R, Wolfson C, Boucher J, Adam J, Hachinski V. The Canadian Neurological Scale: Validation and reliability assessment. Neurology. 1989;39: 638-43.
33. Brott T, Adams Jr HP, Olinger CP, Marler JR, Barsan WG, Biller J, Spilker J, Holleran R, Eberle R, Hertzberg V, et al. Measurements of acute cerebral infarction: a clinical examination scale. Stroke. 1989;20(7):864-70.

34. Alexander M. Stroke rehabilitation outcome: a potential use of predictive variables to establish levels of care. Stroke. 1994;25(1):128-34.

35. Langhorne P, Taylor G, Murray G, Dennis M, Anderson C, Bautz-Holter E, Dey P, Indredavik B, Mayo N, Power M, et al. Early supported discharge services for stroke patients: a meta-analysis of individual patients' data. Lancet. 2005;365(9458):501-6.

36. Kwakkel G, van Peppen R, Wagenaar RC, Wood Dauphinee S, Richards C, Ashburn A, Miller K, Lincoln N, Partridge C, Wellwood I, et al. Effects of augmented exercise therapy time after stroke: a meta-analysis. Stroke. 2004; 35(11):2529-39.

37. Smith J, Forster A, Young J, Cochrane Group for information provision after stroke. Cochrane review: information provision for stroke patients and their caregivers. Clin Rehabil. 2009;23(3):195-206.

\section{Submit your next manuscript to BioMed Central and we will help you at every step:}

- We accept pre-submission inquiries

- Our selector tool helps you to find the most relevant journal

- We provide round the clock customer support

- Convenient online submission

- Thorough peer review

- Inclusion in PubMed and all major indexing services

- Maximum visibility for your research

Submit your manuscript at www.biomedcentral.com/submit
Biomed Central 